\title{
A Nontrivial Product of Filtration $s+5$ in the Stable Homotopy of Spheres
}

\author{
Xiu Gui LIU \\ Department of Mathematics and LPMC, Nankai University, Tianjin 300071, P. R. China \\ E-mail: mathelxg@yahoo.com.cn
}

\begin{abstract}
In this paper, some groups $\operatorname{Ext}_{A}^{s, t}\left(Z_{p}, Z_{p}\right)$ with specialized $s$ and $t$ are first computed by the May spectral sequence. Then we make use of the Adams spectral sequence to prove the existence of a new nontrivial family of filtration $s+5$ in the stable homotopy groups of spheres $\pi_{p^{n} q+(s+3) p q+(s+1) q-5} S$ which is represented (up to a nonzero scalar) by $\tilde{\beta}_{s+2} b_{0} h_{n} \in \operatorname{Ext}_{A}^{s+5, p^{n} q+(s+3) p q+(s+1) q+s}\left(Z_{p}, Z_{p}\right)$ in the Adams spectral sequence, where $p \geq 5$ is a prime number, $n \geq 3,0 \leq s<p-3, q=2(p-1)$.
\end{abstract}

Keywords Stable homotopy of spheres, Adams spectral sequence, Toda-Smith spectrum, May spectral sequence

MR(2000) Subject Classification 55Q45, 55T15

\section{Introduction}

Let $A$ be the mod $p$ Steenrod algebra and $S$ be the sphere spectrum localized at an odd prime number $p$. To determine the stable homotopy groups of spheres $\pi_{*} S$ is one of the central problems in homotopy theory. One of the main tools to reach it is the Adams spectral sequence (ASS) $E_{2}^{s, t}=\operatorname{Ext}_{A}^{s, t}\left(Z_{p}, Z_{p}\right) \Rightarrow \pi_{t-s} S$, where the $E_{2}^{s, t}$-term is the cohomology of $A$. If a family of homotopy generators $x_{i}$ in $E_{2}^{s, *}$ converges nontrivially in the ASS, then we get a family of homotopy elements $f_{i}$ in $\pi_{*} S$ and we say that $f_{i}$ is represented by $x_{i} \in E_{2}^{s, *}$ and has filtration $s$ in the ASS. So far, not so many families of homotopy elements in $\pi_{*} S$ have been detected. For example, a family $\zeta_{n-1} \in \pi_{p^{n} q+q-3} S$ for $n \geq 2$ which has filtration 3 in the ASS and is represented by $h_{0} b_{n-1} \in \operatorname{Ext}_{A}^{3, p^{n} q+q}\left(Z_{p}, Z_{p}\right)$ has been detected in reference [1], where $q=2(p-1)$. In this paper, we detect a family of homotopy elements in $\pi_{*} S$ which has filtration $s+5$ in the ASS.

From reference [2], $\operatorname{Ext}_{A}^{1, *}\left(Z_{p}, Z_{p}\right)$ has $Z_{p}$-bases consisting of $a_{0} \in \operatorname{Ext}_{A}^{1,1}\left(Z_{p}, Z_{p}\right), h_{i} \in$ $\operatorname{Ext}_{A}^{1, p^{i} q}\left(Z_{p}, Z_{p}\right)$ for all $i \geq 0$ and $\operatorname{Ext}_{A}^{2, *}\left(Z_{p}, Z_{p}\right)$ has $Z_{p}$-bases consisting of $\alpha_{2}, a_{0}^{2}, a_{0} h_{i}(i>0)$, $g_{i}(i \geq 0), k_{i}(i \geq 0), b_{i}(i \geq 0)$, and $h_{i} h_{j}(j \geq i+2, i \geq 0)$ whose internal degrees are $2 q+1,2$, $p^{i} q+1, p^{i+1} q+2 p^{i} q, 2 p^{i+1} q+p^{i} q, p^{i+1} q$ and $p^{i} q+p^{j} q$, respectively.

Let $M$ be the Moore spectrum modulo a prime number $p \geq 3$ given by the cofibration

$$
S \stackrel{p}{\rightarrow} S \stackrel{i}{\rightarrow} M \stackrel{j}{\rightarrow} \Sigma S
$$

Let $\alpha: \Sigma^{q} M \rightarrow M$ be the Adams map and $K$ be its cofibre given by the cofibration

$$
\Sigma^{q} M \stackrel{\alpha}{\rightarrow} M \stackrel{i^{\prime}}{\rightarrow} K \stackrel{j^{\prime}}{\rightarrow} \Sigma^{q+1} M
$$

where $q=2(p-1)$. This spectrum which we write in brief as $K$ is known to be the Toda-Smith spectrum $V(1)$. Let $V(2)$ be the cofibre of $\beta: \Sigma^{(p+1) q} K \rightarrow K$ given by the cofibration

$$
\Sigma^{(p+1) q} K \stackrel{\beta}{\rightarrow} K \stackrel{\bar{i}}{\rightarrow} V(2) \stackrel{\bar{j}}{\rightarrow} \Sigma^{(p+1) q+1} K .
$$

Received September 15, 2003, Accepted June 28, 2004

This paper is supported by the National Natural Science Foundation of China (No. 10501045, 10426028), the China Postdoctoral Science Foundation and the Fund of the Personnel Division of Nankai University 
As we know, in the classical Adams spectral sequence the $\beta$-element $\beta_{t}=j j^{\prime} \beta^{t} i^{\prime} i$ is a nontrivial element of order $p$ in $\pi_{(p+1) t q-q-2} S$, where $p \geq 5$.

In this paper, we will prove the following theorem.

Theorem 1.1 Let $p \geq 5, n \geq 3$. Then $\tilde{\beta}_{s+2} b_{0} h_{n} \neq 0 \in \operatorname{Ext}_{A}^{s+5, p^{n} q+(s+3) p q+(s+1) q+s}\left(Z_{p}, Z_{p}\right)$ is a permanent cycle in the Adams Spectral Sequence and converges to a nontrivial element in $\pi_{p^{n} q+(s+3) p q+(s+1) q-5}$, where $0 \leq s<p-3, q=2(p-1)$.

Remark The $\tilde{\beta}_{s+2} b_{0} h_{n}$-element obtained in Theorem 1.1 actually is the product of the $\beta$-element $\beta_{s+2}=j j^{\prime} \beta^{s+2} i^{\prime} i \in \pi_{(s+2)(p+1) q-q-2} S$ and the $\left(b_{0} h_{n}+h_{1} b_{n-1}\right)$-element $j \xi_{n} \in$ $\pi_{p^{n} q+p q-3} S$ in reference [3].

After giving some preliminaries on Ext groups of lower dimension in Section 2, the proof of Theorem 1.1 will be given in Section 3 .

\section{Some Preliminaries on Ext groups}

In this section, we will first prove some results on Ext groups of lower dimension which will be used in the proof of the main theorem.

From $\left[4\right.$, Theorem 3.2.5], there is a May spectral sequence (MSS) $\left\{E_{r}^{s, t, *}, d_{r}\right\}$ which converges to $\operatorname{Ext}_{A}^{s, t}\left(Z_{p}, Z_{p}\right)$ with $E_{1}$-term

$$
E_{1}^{*, *, *}=E\left(h_{m, i} \mid m>0, i \geq 0\right) \otimes P\left(b_{m, i} \mid m>0, i \geq 0\right) \otimes P\left(a_{n} \mid n \geq 0\right),
$$

where $E$ is the exterior algebra, $P$ is the polynomial algebra, and $h_{m, i} \in E_{1}^{1,2\left(p^{m}-1\right) p^{i}, 2 m-1}$, $b_{m, i} \in E_{1}^{2,2\left(p^{m}-1\right) p^{i+1}, p(2 m-1)}, a_{n} \in E_{1}^{1,2 p^{n}-1,2 n+1}$. One has $d_{r}: E_{r}^{s, t, u} \rightarrow E_{r}^{s+1, t, u-r}$ and if $x \in E_{r}^{s, t, *}, y \in E_{r}^{s^{\prime}, t^{\prime}, *}$, then $d_{r}(x \cdot y)=d_{r}(x) \cdot y+(-1)^{s} x \cdot d_{r}(y) . \quad x y=(-1)^{s s^{\prime}+t t^{\prime}} y x$ for $x, y=h_{m, i}, b_{m, i}$ or $a_{n}$. The first May differential $d_{1}$ is given by

$$
d_{1}\left(h_{i, j}\right)=\sum_{0<k<i} h_{i-k, k+j} h_{k, j}, \quad d_{1}\left(a_{i}\right)=\sum_{0 \leq k<i} h_{i-k, k} a_{k}, \quad d_{1}\left(b_{i, j}\right)=0 .
$$

For any element $x \in E_{1}^{s, t, *}$, define $\operatorname{dim} x=s, \operatorname{deg} x=t$. Then we have

$$
\begin{aligned}
& \operatorname{dim} h_{i, j}=\operatorname{dim} a_{i}=1, \quad \operatorname{dim} b_{i, j}=2, \\
& \operatorname{deg} h_{i, j}=2\left(p^{i}-1\right) p^{j}=2(p-1)\left(p^{i+j-1}+\cdots+p^{j}\right), \\
& \operatorname{deg} b_{i, j}=2\left(p^{i}-1\right) p^{j+1}=2(p-1)\left(p^{i+j}+\cdots+p^{j+1}\right), \\
& \operatorname{deg} a_{i}=2 p^{i}-1=2(p-1)\left(p^{i-1}+\cdots+1\right)+1, \\
& \operatorname{deg} a_{0}=1,
\end{aligned}
$$

where $i \geq 1, j \geq 0$.

Lemma 2.1 Let $t=q\left(c_{n} p^{n}+c_{n-1} p^{n-1}+\cdots+c_{1} p+c_{0}\right)+e$ be a positive integer with $0 \leq c_{i}<p$ $(0 \leq i \leq n), 0 \leq e<q$, s be a positive integer with $0<s<p$. If for some $j(0 \leq j \leq n)$, $s<c_{j}$, then in the MSS we have $E_{1}^{s, t, *}=0$.

Proof Suppose that $h=x_{1} x_{2} \cdots x_{m}$ is the generator of $E_{1}^{s, t, *}$, where $x_{i}$ is one of $a_{k}, h_{l, j}$ or $b_{u, z}, 0 \leq k \leq n+1,0 \leq l+j \leq n+1,0 \leq u+z \leq n, l>0, j \geq 0, u>0, z \geq 0$. Assume that $\operatorname{deg} x_{i}=q\left(a_{i, n} p^{n}+\cdots+a_{i, 1} p+a_{i, 0}\right)+e_{i}$, where $a_{i, j}=0$ or $1, e_{i}=1$ if $x_{i}=a_{k_{i}}$, or $e_{i}=0$. Then

$$
\begin{aligned}
\operatorname{deg} h & =\sum_{i=1}^{m} \operatorname{deg} x_{i} \\
& =q\left(\left(\sum_{i=1}^{m} a_{i, n}\right) p^{n}+\cdots\left(\sum_{i=1}^{m} a_{i, 1}\right) p+\left(\sum_{i=1}^{m} a_{i, 0}\right)\right)+\left(\sum_{i=1}^{m} e_{i}\right) \\
& =q\left(c_{n} p^{n}+c_{n-1} p^{n-1}+\cdots+c_{1} p+c_{0}\right)+e,
\end{aligned}
$$




$$
\operatorname{dim} h=\sum_{i=1}^{m} \operatorname{dim} x_{i}=s .
$$

By the facts that $\operatorname{dim} h_{i, j}=\operatorname{dim} a_{i}=1$ and $\operatorname{dim} b_{i, j}=2$, we know that $0<m \leq s<p$ from the equality $\sum_{i=1}^{m} \operatorname{dim} x_{i}=s$. Noting that $a_{i, j}=0$ or $1, e_{i}=0$ or 1 and $m<p$, we have: $\sum_{i=1}^{m} e_{i}=e, \sum_{i=1}^{m} a_{i, 0}=c_{0}, \sum_{i=1}^{m} a_{i, 1}=c_{1}, \ldots, \sum_{i=1}^{m} a_{i, j-1}=c_{j-1}, \sum_{i=1}^{m} a_{i, j}=c_{j}, \ldots$, $\sum_{i=1}^{m} a_{i, n}=c_{n}$. Noting the supposition that $a_{i, j}=0$ or 1 , from the equality $\sum_{i=1}^{m} a_{i, j}=c_{j}$, we have $m \geq c_{j}$. But we also know that $c_{j}>s$, so $m>s$. Therefore we have $s \geq m>s$. That is impossible. This finishes the proof of Lemma 2.1.

Theorem 2.2 For $p \geq 5,0 \leq s<p-2$, the element

$$
\underbrace{a_{2} a_{2} \cdots a_{2}}_{s} h_{2,0} h_{1,1} \in E_{r}^{s+2,(s+2) p q+(s+1) q+s, *}
$$

in the May spectral sequence converges to the second Greek letter family element $\tilde{\beta}_{s+2} \in$ $\operatorname{Ext}_{A}^{s+2, t}\left(Z_{p}, Z_{p}\right)$, where $r \geq 1, t=(s+2) p q+(s+1) q+s$, and $\tilde{\beta}_{s+2}$ converges to the $\beta$ element $\beta_{s+2} \in \pi_{(s+2) p q+(s+1) q-2} S$ in the Adams spectral sequence.

Proof From [5, Theorems 1 and 2], we know that the $\beta$-element $\beta_{s+2} \in \pi_{(s+2) p q+(s+1) q-2} S$ is represented by the second Greek letter family element $\tilde{\beta}_{s+2} \in \operatorname{Ext}_{A}^{s+2, t}\left(Z_{p}, Z_{p}\right)$ in the ASS, where $t=(s+2) p q+(s+1) q+s$. However in the MSS, $E_{1}^{s+2,(s+2) p q+(s+1) q+s, *}=$ $Z_{p}\{\underbrace{a_{2} a_{2} \cdots a_{2}}_{s} h_{2,0} h_{1,1}\}$ (This will be proved later.), so in the MSS, $\tilde{\beta}_{s+2} \in \operatorname{Ext}_{A}^{s+2, t}\left(Z_{p}, Z_{p}\right)$ is represented by $\underbrace{a_{2} a_{2} \cdots a_{2}}_{s} h_{2,0} h_{1,1} \in E_{1}^{s+2, t, *}$.

Now our remaining work is to prove $E_{1}^{s+2, t, *}=Z_{p}\{\underbrace{a_{2} a_{2} \cdots a_{2}}_{s} h_{2,0} h_{1,1}\}$. Suppose that $h=x_{1} x_{2} \cdots x_{m}$ is the generator of $E_{1}^{s+2, t, *}$, where $x_{i}$ is one of $a_{k}, h_{l, j}$ or $b_{u, z}, 0 \leq k \leq 2$, $0 \leq l+j \leq 2,0 \leq u+z \leq 1, l>0, j \geq 0, u>0, z \geq 0$. Assume that deg $x_{i}=q\left(a_{i, 1} p+a_{i, 0}\right)+e_{i}$, where $a_{i, j}=0$ or $1, e_{i}=1$ if $x_{i}=a_{k_{i}}$, or $e_{i}=0$. Then

$$
\begin{aligned}
\operatorname{deg} h & =\sum_{i=1}^{m} \operatorname{deg} x_{i} \\
& =q\left(\left(\sum_{i=1}^{m} a_{i, 1}\right) p+\left(\sum_{i=1}^{m} a_{i, 0}\right)\right)+\left(\sum_{i=1}^{m} e_{i}\right) \\
& =q((s+2) p+(s+1))+s, \\
\operatorname{dim} h & =\sum_{i=1}^{m} \operatorname{dim} x_{i}=s+2 .
\end{aligned}
$$

By the facts that $\operatorname{dim} h_{i, j}=\operatorname{dim} a_{i}=1$ and $\operatorname{dim} b_{i, j}=2$, we know that $0<m \leq s+2$ from $\sum_{i=1}^{m} \operatorname{dim} x_{i}=s+2$. Noting that $a_{i, j}=0$ or $1, e_{i}=0$ or 1 and $m \leq s+2<p$, we have: $\sum_{i=1}^{m} e_{i}=s, \sum_{i=1}^{m} a_{i, 0}=s+1$ and $\sum_{i=1}^{m} a_{i, 1}=s+2$. From the equality $\sum_{i=1}^{m} a_{i, 1}=s+2$ and the fact that $a_{i, 2}=0$, or $a_{i, 2}=1$, we see that $m \geq s+2$, so $m=s+2$. Since $\operatorname{dim} h=\sum_{i=1}^{s+2} \operatorname{dim} x_{i}=s+2$, then for any $1 \leq i \leq s+2, \operatorname{dim} x_{i}=1$, so we get that $h \in P\left(a_{n} \mid n \geq 0\right) \otimes E\left(h_{m, i} \mid m>0, i \geq 0\right)$.

Since $\sum_{i=1}^{s+2} e_{i}=s \equiv s(\bmod q), \operatorname{deg} h_{i, j} \equiv 0(\bmod q)(i>0, j \geq 0)$ and $\operatorname{deg} a_{i} \equiv 1(\bmod q)$ $(i \geq 0)$, then the generator $h$ must have a factor $a_{j_{1}} a_{j_{2}} \cdots a_{j_{s}}$. Noting the degrees of $a_{i}$ 's and the commutativity of $E_{1}^{*, *, *}$, we can suppose that $h=\underbrace{a_{0} \cdots a_{0}}_{x} \underbrace{a_{1} \cdots a_{1}}_{y} \underbrace{a_{2} \cdots a_{2}}_{z} x_{s+1} x_{s+2}$

(up to sign), where $0 \leq x, y, z \leq s, x+y+z=s$. Then we get that $x+y+z+\sum_{i=s+1}^{s+2} e_{i}=s$, $y+z+\sum_{i=s+1}^{s+2} a_{i, 0}=s+1$ and $z+\sum_{i=s+1}^{s+2} a_{i, 1}=s+2$. From the equality $z+\sum_{i=s+1}^{s+2} a_{i, 1}=s+2$, 
we can get that $z=s+2-\sum_{i=s+1}^{s+2} a_{i, 1} \geq s+2-2=s$. So $z=s, x=y=0$, that is, $h=\underbrace{a_{2} a_{2} \cdots a_{2}}_{s} x_{s+1} x_{s+2}$. It is easy to show that $x_{s+1} x_{s+2} \in E_{1}^{2,2 p q+q, *} \cong Z_{p}\left\{h_{2,0} h_{1,1}\right\}$. It follows that $h=\underbrace{a_{2} a_{2} \cdots a_{2}}_{s} h_{2,0} h_{1,1}$ (up to sign) and $E_{1}^{s+2, t, *}=Z_{p}\{\underbrace{a_{2} a_{2} \cdots a_{2}}_{s} h_{2,0} h_{1,1}\}$.

Proposition 2.3 Let $p \geq 5, n \geq 3,0 \leq s<p-3$. Then

$$
\tilde{\beta}_{s+2} b_{0} h_{n} \neq 0 \in \operatorname{Ext}_{A}^{s+5, p^{n} q+(s+3) p q+(s+1) q+s}\left(Z_{p}, Z_{p}\right) .
$$

Proof First consider the structure of $E_{1}^{s+4, t^{\prime}, *}$ in the MSS, where $t^{\prime}=p^{n} q+(s+3) p q+(s+1) q+s$. Since $0 \leq s<p-3$, then $4 \leq s+4<p+1$. Suppose that $h=x_{1} x_{2} \cdots x_{m}$ is the generator of $E_{1}^{s+4, t^{\prime}, *}$, where $x_{i}$ is one of $a_{k}, h_{l, j}$ or $b_{u, z}, 0 \leq k \leq n+1,0 \leq l+j \leq n+1,0 \leq u+z \leq n$, $l>0, j \geq 0, u>0, z \geq 0 . \operatorname{deg} x_{i}=q\left(a_{i, n} p^{n}+a_{i, n-1} p^{n-1}+\cdots+a_{i, 0}\right)+e_{i}$, where $a_{i, j}=0$ or $1, e_{i}=1$ if $x_{i}=a_{k_{i}}$, or $e_{i}=0$. Then

$$
\begin{aligned}
\operatorname{deg} h & =\sum_{i=1}^{m} \operatorname{deg} x_{i} \\
& =q\left(\left(\sum_{i=1}^{m} a_{i, n}\right) p^{n}+\cdots+\left(\sum_{i=1}^{m} a_{i, 2}\right) p^{2}+\left(\sum_{i=1}^{m} a_{i, 1}\right) p+\left(\sum_{i=1}^{m} a_{i, 0}\right)\right)+\left(\sum_{i=1}^{m} e_{i}\right) \\
& =q\left(p^{n}+(s+3) p+(s+1)\right)+s, \\
\operatorname{dim} h & =\sum_{i=1}^{m} \operatorname{dim} x_{i}=s+4 .
\end{aligned}
$$

Noting that $\operatorname{dim} x_{i}=1$ or 2 , we have $m \leq s+4 \leq p$ from $\sum_{i=1}^{m} \operatorname{dim} x_{i}=s+4$. By the knowledge about the $p$-adic expression in number theory and the suppositions that $a_{i, j}=0$ or $a_{i, j}=1, e_{i}=0$ or $1, m \leq s+4 \leq p$, we have

$$
\begin{aligned}
& \sum_{i=1}^{m} e_{i}=s, \quad \sum_{i=1}^{m} a_{i, 0}=s+1, \\
& \sum_{i=1}^{m} a_{i, 1}=s+3, \quad\left(\sum_{i=1}^{m} a_{i, 2}\right) p^{2}+\left(\sum_{i=1}^{m} a_{i, 3}\right) p^{3}+\cdots+\left(\sum_{i=1}^{m} a_{i, n}\right) p^{n}=p^{n} .
\end{aligned}
$$

Case $10 \leq s<p-4$.

By the knowledge about the $p$-adic expression in number theory and (o), we have

$$
\begin{aligned}
& \sum_{i=1}^{m} e_{i}=s, \quad \sum_{i=1}^{m} a_{i, 0}=s+1, \quad \sum_{i=1}^{m} a_{i, 1}=s+3, \\
& \sum_{i=1}^{m} a_{i, 2}=\cdots=\sum_{i=1}^{m} a_{i, n-1}=0, \quad \sum_{i=1}^{m} a_{i, n}=1 .
\end{aligned}
$$

It easy to see that there exists a factor $h_{1, n}$ or $b_{1, n-1}$ among $x_{i}$ 's. By the commutativity of $E_{1}^{*, *, *}$, we can denote $h_{1, n}$ or $b_{1, n-1}$ by $x_{m}$.

If $h=x_{1} x_{2} \cdots x_{m-1} h_{1, n}, h^{\prime}=x_{1} x_{2} \cdots x_{m-1} \in E_{1}^{s+3, t^{\prime}-p^{n} q, *}$. By an argument similar to that used in the proof of Theorem 2.2, we can show that $E_{1}^{s+3, t^{\prime}-p^{n} q, *}=0$, so $h^{\prime}$ is impossible to exist. Thus $h$ is impossible to be of the form $h=x_{1} x_{2} \cdots x_{m-1} h_{1, n}$.

If $h=x_{1} x_{2} \cdots x_{m-1} b_{1, n-1}, h^{\prime \prime}=x_{1} x_{2} x_{3} \cdots x_{m-1} \in E_{1}^{s+2, t^{\prime}-p^{n} q, *}$. By Lemma 2.1, we can know that $E_{1}^{p-2, t^{\prime}-p^{n} q, *}=0$, so $h^{\prime \prime}=x_{1} x_{2} x_{3} \cdots x_{m-1}$ is impossible to exist and $h$ is impossible to be of the form $h=x_{1} x_{2} \cdots x_{m-1} b_{1, n-1}$.

From the above discussion, we see that when $0 \leq s<p-4=0, E_{1}^{s+4, t^{\prime}, *}=0$. Thus $E_{r}^{s+4, t^{\prime}, *}=0$ for $r \geq 1$. It is known that $h_{1, n}, b_{1, n}, \underbrace{a_{2} a_{2} \cdots a_{2}}_{s} h_{2,0} h_{1,1} \in E_{1}^{*, *, *}$ are permanent 
cycles in the MSS and converge nontrivially to $h_{n}, b_{n}, \tilde{\beta}_{s+2} \in \operatorname{Ext}_{A}^{* * *}\left(Z_{p}, Z_{p}\right)$ for $n \geq 0$, respectively (cf. Theorem 2.2), so at this time the permanent cycle $\underbrace{a_{2} a_{2} \cdots a_{2}}_{s} h_{2,0} h_{1,1} b_{1,0} h_{1, n} \in$ $E_{r}^{s+5, t^{\prime}, *}$ is not bounded and converges nontrivially to $\tilde{\beta}_{s+2} b_{0} h_{n} \in \operatorname{Ext}_{A}^{s+5, t^{\prime}}\left(Z_{p}, Z_{p}\right)$ in the MSS. Thus $\tilde{\beta}_{s+2} b_{0} h_{n} \neq 0 \in \operatorname{Ext}_{A}^{s+5, t^{\prime}}\left(Z_{p}, Z_{p}\right)$.

Case $2 s=p-4$

$E_{1}^{s+4, t^{\prime}, *}=E_{1}^{p, t^{\prime \prime}, *}$, where $t^{\prime \prime}=p^{n} q+(p-1) p q+(p-3) q+(p-4)$. Noting that $m \leq s+4=p$, from (\$) we have

then

$$
\left(\sum_{i=1}^{m} a_{i, 2}\right) p^{2}+\left(\sum_{i=1}^{m} a_{i, 3}\right) p^{3}+\cdots+\left(\sum_{i=1}^{m} a_{i, n}\right) p^{n}=p^{n}
$$

$$
\left(\sum_{i=1}^{m} a_{i, 2}\right)+\left(\sum_{i=1}^{m} a_{i, 3}\right) p+\cdots+\left(\sum_{i=1}^{m} a_{i, n}\right) p^{n-2}=p^{n-2},
$$

so $p \mid \sum_{i=1}^{m} a_{i, 2}$. Note that $a_{i, 2}=0$ or $1, m \leq p$. It is easy to know that $\sum_{i=1}^{m} a_{i, 2}=0$ or $\sum_{i=1}^{m} a_{i, 2}=p$.

Subcase $2.1 \quad \sum_{i=1}^{m} a_{i, 2}=0$.

If $n=3$, it is easy to get that $\sum_{i=1}^{m} a_{i, 3}=1$, so there exists a factor $h_{1, n}$ or $b_{1, n-1}$ among $x_{i}$ 's.

If $n>3$, then $\left(\sum_{i=1}^{m} a_{i, 3}\right) p^{3}+\left(\sum_{i=1}^{m} a_{i, 4}\right) p^{4}+\cdots+\left(\sum_{i=1}^{m} a_{i, n}\right) p^{n}=p^{n}$, so

$$
\left(\sum_{i=1}^{m} a_{i, 3}\right)+\left(\sum_{i=1}^{m} a_{i, 4}\right) p+\left(\sum_{i=1}^{m} a_{i, 5}\right) p^{2}+\cdots+\left(\sum_{i=1}^{m} a_{i, n}\right) p^{n-3}=p^{n-3} .
$$

Similarly we know that $\sum_{i=1}^{m} a_{i, 3}=0$ or $\sum_{i=1}^{m} a_{i, 3}=p$. We claim that $\sum_{i=1}^{m} a_{i, 3}=0$, for otherwise, we would have $\sum_{i=1}^{m} a_{i, 3}=p$, then $m=p$. Then for each $1 \leq i \leq p$, $\operatorname{dim} x_{i}=1$ and $\operatorname{deg} x_{i}=$ higher terms $+p^{3} q+$ lower terms. Since $\sum_{i=1}^{p} e_{i}=p-4, \operatorname{deg} a_{i} \equiv 1(\bmod q)(i \geq 0)$ and $\operatorname{deg} h_{i, j} \equiv 0(\bmod q)(i>0, j \geq 0)$, then there exists a factor $a_{j_{1}} a_{j_{2}} \cdots a_{j_{p-4}}$ among $x_{i}$ 's such that for each $1 \leq i \leq p-4, j_{i} \geq 4$ and $\operatorname{deg} a_{j_{i}}=$ higher terms $+p^{3} q+p^{2} q+p q+q+1$. It is obvious that $\sum_{i=1}^{m} a_{i, 2} \geq p-4$ which contradicts $\sum_{i=1}^{m} a_{i, 2}=0$, thus the claim follows. By induction on $j$, we can get that $\sum_{i=1}^{m} a_{i, j}=0(3 \leq j \leq n-1)$, so $\sum_{i=1}^{m} a_{i, n}=1$, that is to say, there is a factor $h_{1, n}$ or $b_{1, n-1}$ among $x_{i}$ 's.

All in all, at this time for $n \geq 3$, there is a factor $h_{1, n}$ or $b_{1, n-1}$ among $x_{i}$ 's. We denote $h_{1, n}$ or $b_{1, n-1}$ by $x_{m}$, then $h=x_{1} x_{2} \cdots x_{m-1} h_{1, n}$ (up to sign) or $h=x_{1} x_{2} \cdots x_{m-1} b_{1, n-1}$ (up to sign).

If $h=x_{1} x_{2} \cdots x_{m-1} h_{1, n}, h^{\prime}=x_{1} x_{2} \cdots x_{m-1} \in E_{1}^{p-1, t^{\prime \prime}-p^{n} q, *}$. By an argument similar to that used in the proof of Theorem 2.2, we can show that $E_{1}^{s+3, t^{\prime \prime}-p^{n} q, *}=0$, so $h^{\prime}$ is impossible to exist. Thus $h$ is impossible to be of the form $h=x_{1} x_{2} \cdots x_{m-1} h_{1, n}$.

If $h=x_{1} x_{2} \cdots x_{m-1} b_{1, n-1}, h^{\prime \prime}=x_{1} x_{2} x_{3} \cdots x_{m-1} \in E_{1}^{p-2, t^{\prime \prime}-p^{n} q, *}$. By Lemma 2.1, we can know that $E_{1}^{p-2, t^{\prime \prime}-p^{n} q, *}=0$, so $h^{\prime \prime}=x_{1} x_{2} x_{3} \cdots x_{m-1}$ is impossible to exist and $h$ is impossible to be of the form $h=x_{1} x_{2} \cdots x_{m-1} b_{1, n-1}$.

Subcase 2.2 If $\sum_{i=1}^{m} a_{i, 2}=p$, then $m=p$. Since $\operatorname{dim} h=p$, we can easily see that for each $i, \operatorname{dim} x_{i}=1$ and $h=x_{1} x_{2} \cdots x_{p} \in E\left(h_{m, i} \mid m>0, i \geq 0\right) \otimes P\left(a_{n} \mid n \geq 0\right)$.

If $n=3$, we can easily get that $\sum_{i=1}^{p} a_{i, 2}=p, \sum_{i=1}^{p} a_{i, 1}=p-1, \sum_{i=1}^{p} a_{i, 0}=p-3$ and $\sum_{i=1}^{p} e_{i}=p-4$.

If $n>3$, from the equality $\left(\sum_{i=1}^{p} a_{i, 2}\right) p^{2}+\cdots+\left(\sum_{i=1}^{p} a_{i, n}\right) p^{n}=p^{n}$, we can have

$$
\left(\sum_{i=1}^{p} a_{i, 3}+1\right)+\left(\sum_{i=1}^{p} a_{i, 4}\right) p+\cdots+\left(\sum_{i=1}^{p} a_{i, n}\right) p^{n}=p^{n} .
$$


Then $p \mid\left(\sum_{i=1}^{p} a_{i, 3}+1\right)$. Noting that $a_{i, 3}=0$ or 1 , we have that $\sum_{i=1}^{p} a_{i, 3}=p-1$. By induction on $j$, we can prove that $\sum_{i=1}^{p} a_{i, j}=p-1(3 \leq j \leq n-1)$. So $\sum_{i=1}^{p} a_{i, n}=0$.

When $n=3$, by the facts that $\sum_{i=1}^{p} e_{i}=p-4, \sum_{i=1}^{p} a_{i, 0}=p-3, \sum_{i=1}^{p} a_{i, 1}=p-1$, $\sum_{i=1}^{p} a_{i, 2}=p$, we can prove that $h=x_{1} x_{2} \cdots x_{p}$ is impossible to exist by an argument similar to that used in the proof of Theorem 2.2 .

When $n>3$, by the facts that $\sum_{i=1}^{p} a_{i, 2}=p, \sum_{i=1}^{p} a_{i, 3}=\cdots=\sum_{i=1}^{p} a_{i, n-1}=p-1$, $\operatorname{deg} h_{k, j}=q\left(p^{k+j-1}+\cdots+p^{j}\right)(k \geq 1, j \geq 0)$ and $\operatorname{deg} a_{i}=q\left(p^{i-1}+\cdots+p+1\right)+1(i>0)$, we can divide the $p x_{i}$ 's into two disjoint classes $S_{1}$ and $S_{2}$. The two disjoint classes are given by

$$
\begin{aligned}
& S_{1}=\left\{x \mid \operatorname{deg} x=q\left(p^{n-1}+p^{n-2}+\cdots+p^{2}\right)+\text { lower terms }\right\}, \\
& S_{2}=\left\{x \mid \operatorname{deg} x=q p^{2}+\text { lower terms }\right\} .
\end{aligned}
$$

For a class $S$, denote the number of elements in $S$ by $N(S)$, then we can get $N\left(S_{1}\right)=p-1$ and $N\left(S_{2}\right)=1$. Similarly, by the facts that $\sum_{i=1}^{p} e_{i}=p-4, \sum_{i=1}^{p} a_{i, 0}=p-3, \sum_{i=1}^{p} a_{i, 1}=p-1$, $\sum_{i=1}^{p} a_{i, 2}=p, \operatorname{deg} h_{k, j}=q\left(p^{k+j-1}+\cdots+p^{j}\right)(k \geq 1, j \geq 0)$ and $\operatorname{deg} a_{i}=q\left(p^{i-1}+\cdots+p+1\right)+1$ $(i>0)$, we can also divide the $p x_{i}$ 's into four disjoint classes. The four classes are given by

$$
\begin{aligned}
& S_{3}=\left\{x \mid \operatorname{deg} x=q\left(\text { higher terms }+p^{2}+p+1\right)+1\right\}, \quad N\left(S_{3}\right)=p-4, \\
& S_{4}=\left\{x \mid \operatorname{deg} x=q\left(\text { higher terms }+p^{2}+p+1\right)\right\}, \quad N\left(S_{4}\right)=1, \\
& S_{5}=\left\{x \mid \operatorname{deg} x=q\left(\text { higher terms }+p^{2}+p\right)\right\}, \quad N\left(S_{5}\right)=2, \\
& S_{6}=\left\{x \mid \operatorname{deg} x=q\left(\text { higher terms }+p^{2}\right)\right\}, \quad N\left(S_{6}\right)=1 .
\end{aligned}
$$

If $S_{5} \subset S_{1}$ (i.e., all elements in $S_{5}$ are in $S_{1}$ ), then there would be two $h_{n-1,1}$ 's such that $\operatorname{deg} h_{n-1,1}=q\left(p^{n-1}+\cdots+p^{3}+p^{2}+p\right)$. This is impossible since $h_{n-1,1}^{2}=0$. If $S_{5} \not \subset S_{1}$ (i.e., not all elements in $S_{5}$ are in $S_{1}$ ), then one of the two elements in $S_{5}$ must be in $S_{2}$. The element must be $h_{2,1}$ such that $\operatorname{deg} h_{2,1}=q\left(p^{2}+p\right)$. For two classes $A$ and $B$, define $A \cup B=\{\mathrm{x} \mid x$ is in $A$ or $x$ is in $B\}$. It follows that $S_{1} \cup S_{2}=S_{3} \cup S_{4} \cup S_{5} \cup S_{6}$, so we have $S_{6} \subset S_{1}, S_{4} \subset S_{1}, S_{3} \subset S_{1}$ and another element in $S_{5}$ must be in $S_{1}$. We easily get that $S_{3}=\{\underbrace{a_{n}, a_{n}, \cdots, a_{n}}_{p-4}\}, S_{4}=\left\{h_{n, 0}\right\}, S_{5}=\left\{h_{2,1}, h_{n-1,1}\right\}$ and $S_{6}=\left\{h_{n-2,2}\right\}$. Thus $h=$ $\underbrace{a_{n} a_{n} \cdots a_{n}}_{p-4} h_{n, 0} h_{n-1,1} h_{n-2,2} h_{2,1}$ (up to sign).

From Subcase 2.1 and Subcase 2.2, we see that when $s=p-4$,

$$
E_{1}^{p, t^{\prime \prime}, *}= \begin{cases}Z_{p}\{\underbrace{a_{n} a_{n} \cdots a_{n}}_{p-4} h_{n, 0} h_{n-1,1} h_{n-2,2} h_{2,1}\}, & \text { if } n>3, \\ 0, & \text { if } n=3 .\end{cases}
$$

When $n=3, E_{r}^{p, t^{\prime \prime}, *}=0 \quad(r \geq 1)$, then $d_{r}\left(E_{r}^{p, t^{\prime \prime}, *}\right)=0$.

When $n>3$, consider the May filtration of elements $\underbrace{a_{2} a_{2} \cdots a_{2}}_{p-4} h_{2,0} h_{1,1} b_{1,0} h_{1, n}$ and $\underbrace{a_{n} a_{n} \cdots a_{n}}_{p-4} h_{n, 0} h_{n-1,1} h_{n-2,2} h_{2,1}$. We see that

$$
\begin{aligned}
& M(\underbrace{a_{2} a_{2} \cdots a_{2}}_{p-4} h_{2,0} h_{1,1} b_{1,0} h_{1, n})=6 p-15=M, \\
& M(\underbrace{a_{n} a_{n} \cdots a_{n}}_{p-4} h_{n, 0} h_{n-1,1} h_{n-2,2} h_{2,1})=(2 n+1) p-2 n-10=M+r \quad \text { with } r>2 .
\end{aligned}
$$

Now

$$
d_{1}(\underbrace{a_{n} a_{n} \cdots a_{n}}_{p-4} h_{n, 0} h_{n-1,1} h_{n-2,2} h_{2,1})=\underbrace{a_{n} a_{n} \cdots a_{n}}_{p-4} h_{n, 0} h_{n-1,1} h_{n-2,2} h_{1,2} h_{1,1}+\cdots \neq 0 .
$$

Thus $E_{2}^{p, t^{\prime \prime}, *}=0$ and no higher May differential hits $\underbrace{a_{2} a_{2} \cdots a_{2}}_{p-4} h_{2,0} h_{1,1} b_{1,0} h_{1, n}$ in the MSS. 
This shows that $\tilde{\beta}_{p-2} b_{0} h_{n} \neq 0 \in \operatorname{Ext}_{A}^{p+1, t^{\prime \prime}}\left(Z_{p}, Z_{p}\right)$.

From Case 1 and Case 2, the proposition follows.

Proposition 2.4 Let $p \geq 5, n \geq 3,0 \leq s<p-3,2 \leq r \leq s+5$. Then we have

$$
\operatorname{Ext}_{A}^{s+5-r, q\left(p^{n}+(s+3) p+(s+1)\right)+(s-r+1)}\left(Z_{p}, Z_{p}\right)=0 .
$$

Proof It suffices to prove that in the MSS $E_{1}^{s+5-r, t^{\prime \prime \prime}, *}=0$, where $t^{\prime \prime \prime}=q\left(p^{n}+(s+3) p+(s+\right.$ $1))+(s-r+1)$. Suppose that $h=x_{1} x_{2} \cdots x_{m}$ is the generator of $E_{1}^{s+5-r, t^{\prime \prime \prime}, *}$, where $x_{i}$ is one of $a_{k}, h_{l, j}$ or $b_{u, z}, 0 \leq k \leq n+1,0 \leq l+j \leq n+1,0 \leq u+z \leq n, l>0, j \geq 0, u>0, z \geq 0$. Let $\operatorname{deg} x_{i}=q\left(a_{i, n} p^{n}+a_{i, n-1} p^{n-1}+\cdots+a_{i, 0}\right)+e_{i}$, where $a_{i, j}=0$ or $1, e_{i}=1$ if $x_{i}=a_{k_{i}}$, or $e_{i}=0$. Then

$$
\begin{aligned}
\operatorname{deg} h & =\sum_{i=1}^{m} \operatorname{deg} x_{i} \\
& =q\left(\left(\sum_{i=1}^{m} a_{i, n}\right) p^{n}+\cdots+\left(\sum_{i=1}^{m} a_{i, 2}\right) p^{2}+\left(\sum_{i=1}^{m} a_{i, 1}\right) p+\left(\sum_{i=1}^{m} a_{i, 0}\right)\right)+\left(\sum_{i=1}^{m} e_{i}\right) \\
& =q\left(p^{n}+(s+3) p+(s+1)\right)+s-r+1, \\
\operatorname{dim} h & =\sum_{i=1}^{m} \operatorname{dim} x_{i}=s+5-r .
\end{aligned}
$$

Noting that $\operatorname{dim} x_{i}=1$ or 2 , we have that $m \leq s+5-r \leq s+3<p$ from $\sum_{i=1}^{m} \operatorname{dim} x_{i}=s+5-r$. We claim that $s-r+1 \geq 0$, otherwise, we would have $p>\sum_{i=1}^{m} e_{i}=q+(s-r+1) \geq q-4 \geq p$. That is impossible. The claim follows.

Noting the suppositions that $a_{i, j}=0$ or $1, e_{i}=0$ or 1 and $m<p$, we have

$$
\begin{aligned}
& \sum_{i=1}^{m} e_{i}=s-r+1, \quad \sum_{i=1}^{m} a_{i, 0}=s+1, \quad \sum_{i=1}^{m} a_{i, 1}=s+3, \\
& \sum_{i=1}^{m} a_{i, 2}=0, \quad \sum_{i=1}^{m} a_{i, 3}=\cdots=\sum_{i=1}^{m} a_{i, n-1}=0, \quad \sum_{i=1}^{m} a_{i, n}=1 .
\end{aligned}
$$

It is easy to see that there exists an $h_{1, n}$ or $b_{1, n-1}$ among $x_{i}$ 's. We denote $h_{1, n}$ or $b_{1, n-1}$ by $x_{m}$, then $h=x_{1} x_{2} \cdots x_{m-1} h_{1, n}$ or $h=x_{1} x_{2} \cdots x_{m-1} b_{1, n-1}$.

If $h=x_{1} x_{2} \cdots x_{m-1} h_{1, n}, h^{\prime}=x_{1} x_{2} \cdots x_{m-1} \in E_{1}^{s+4-r, t^{\prime \prime \prime}-p^{n} q, *}=0$ by Lemma 2.1. Thus $h$ is impossible to be of the form $h=x_{1} x_{2} \cdots x_{m-1} h_{1, n}$.

Similarly, we can show that $h$ is impossible to be of the form $h=x_{1} x_{2} \cdots x_{m-1} b_{1, n-1}$ by Lemma 2.1.

From the above discussion we see that $E_{1}^{s+5-r, t^{\prime \prime \prime}, *}=0$, so $\operatorname{Ext}_{A}^{s+5-r, t^{\prime \prime \prime}}\left(Z_{p}, Z_{p}\right)=0$. This finishes the proof of Proposition 2.4.

Proposition 2.5 Let $p \geq 5, n \geq 3,0 \leq s<s-3$. Then we have

$$
\tilde{\beta}_{s+2} h_{1} b_{n-1}=0 \in \operatorname{Ext}_{A}^{s+5, p^{n} q+(s+3) p q+(s+1) q+s}\left(Z_{p}, Z_{p}\right) \text {. }
$$

Proof Since $h_{1,1}^{2}=0 \in E_{1}^{2,2 p q, *}$, then

$$
\underbrace{a_{2} \cdots a_{2}}_{s} h_{2,0} h_{1,1} h_{1,1} b_{1, n-1}=0 \in E_{1}^{s+5, p^{n} q+(s+3) p q+(s+1) q+s, *},
$$

so $\tilde{\beta}_{s+2} h_{1} b_{n-1}=0 \in \mathrm{Ext}_{A}^{s+5, p^{n} q+(s+3) p q+(s+1) q+s}\left(Z_{p}, Z_{p}\right)$.

\section{Proof of Theorem $\mathbf{1 . 1}$}

In this section, we will give the proof of Theorem 1.1.

Theorem 1.1 Let $p \geq 5, n \geq 3$. Then

$$
\tilde{\beta}_{s+2} b_{0} h_{n} \neq 0 \in \operatorname{Ext}_{A}^{s+5, p^{n} q+(s+3) p q+(s+1) q+s}\left(Z_{p}, Z_{p}\right)
$$


is a permanent cycle in the Adams Spectral Sequence and converges to a nontrivial element in $\pi_{p^{n} q+(s+3) p q+(s+1) q-5}$, where $0 \leq s<p-3, q=2(p-1)$.

Proof From [3, Theorem A], we get that $i_{*}\left(h_{1} h_{n}\right) \in \operatorname{Ext}_{A}^{2, p^{n} q+p q}\left(H^{*} M, Z_{p}\right)$ is a permanent cycle in the ASS and converges to a nontrivial element $\xi \in \pi_{p^{n} q+p q-2} M$. At the same time $j \xi_{n} \in \pi_{p^{n} q+p q-3} S$ is a nontrivial element of order $p$ which is represented (up to a nonzero scalar) by $\left(b_{0} h_{n}+h_{1} b_{n-1}\right) \in \operatorname{Ext}_{A}^{3, p^{n} q+p q}\left(Z_{p}, Z_{p}\right)$ in the ASS.

Consider the following composition of maps:

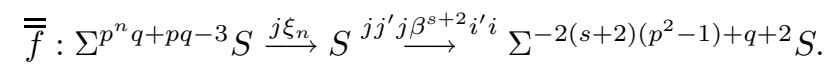

Since $j \xi_{n}$ is represented (up to a nonzero scalar) by $\left(b_{0} h_{n}+h_{1} b_{n-1}\right) \in \operatorname{Ext}_{A}^{3, p^{n} q+p q}\left(Z_{p}, Z_{p}\right)$, then the above $\overline{\bar{f}}$ is represented (up to a nonzero scalar) by $\overline{\bar{c}}=\left(j j^{\prime} \beta^{s+2} i^{\prime} i\right)_{*}\left(b_{0} h_{n}+h_{1} b_{n-1}\right)$.

From Theorem 2.2 and the knowledge of Yoneda products we know that the composition

$$
\operatorname{Ext}_{A}^{0,0}\left(Z_{p}, Z_{p}\right) \stackrel{\left(i^{\prime} i\right)_{*}}{\longrightarrow} \operatorname{Ext}_{A}^{0,0}\left(H^{*} M, Z_{p}\right) \stackrel{\left(j j^{\prime}\right)_{*}\left(\beta_{*}\right)^{s+2}}{\longrightarrow} \operatorname{Ext}_{A}^{s+2,(s+2) p q+(s+1) q+s}\left(Z_{p}, Z_{p}\right)
$$

is a multiplication (up to a nonzero scalar) by $\tilde{\beta}_{s+2} \in \operatorname{Ext}_{A}^{s+2,(s+2) p q+(s+1) q+s}\left(Z_{p}, Z_{p}\right)$. Hence, $\overline{\bar{f}}$ is represented (up to a nonzero scalar) by $\overline{\bar{c}}=\tilde{\beta}_{s+2}\left(b_{0} h_{n}+h_{1} b_{n-1}\right)=\tilde{\beta}_{s+2} b_{0} h_{n} \neq 0 \in$ $\operatorname{Ext}_{A}^{\left.s+5, q\left(p^{n}+(s+3) p+(s+1)\right)+s\right)}\left(Z_{p}, Z_{p}\right)=0$ in the ASS (cf. Proposition 2.3 and Proposition 2.5).

Moreover, from Proposition 2.4, $\operatorname{Ext}_{A}^{s+5-r, q\left(p^{n}+(s+3) p+(s+1)\right)+(s-r+1)}\left(Z_{p}, Z_{p}\right)=0$ for $r \geq 2$, then $\tilde{\beta}_{s+2} b_{0} h_{n}$ cannot be hit by the differentials in the ASS, and so the corresponding homotopy element $\overline{\bar{f}} \in \pi_{*} S$ is nontrivial and of order $p$. This finishes the proof of Theorem 1.1.

\section{References}

[1] Cohen, Ralph L.: Odd primary families in stable homotopy theory. Mem. Amer. Math. Soc., 242, 1-92 (1981)

[2] Liulevicius, A.: The factorizations of cyclic reduced powers by secondary cohomology operations. Mem. Amer. Math. Soc., 42, 1-112 (1962)

[3] Lin, J. K.: A new family of filtration three in the stable homotopy of spheres. Hiroshima Math. J., 31(3), 477-492 (2001)

[4] Ravenel, D. C.: Complex Cobordism and Stable Homotopy Groups of Spheres, Academic Press, Orlando, 1986

[5] Wang, X., Zheng, Q.: The convergence of $\tilde{\alpha}_{s}^{(n)} h_{0} h_{k}$. Sci. China Ser. A, 41(6), $622-628$ (1998)

[6] Cohen, Ralph L., Goerss, P.: Secondary cohomology operations that detect homotopy classes. Topology, 23, 177-194 (1984)

[7] Oka, S.: Multilicative structure of finite ring spectra and stable homotopy of spheres. Algebraic Topology (Aarhus), Lecture Notes in Math., Springer-Verlag, 1051, 418-441, 1984

[8] Zhou, X. G.: Higher cohomology operations that detect homotopy classes, Lecture Notes in Math., SpringerVerlag, 1370, 416-436, 1989

[9] Toda, H.: Algebra of stable homotopy of $Z_{p}$-spaces and applications. J. Math. Kyoto Univ., 11, 197-251 (1971)

[10] Toda, H.: On spectra realizing exterior parts of the Steenrod algebra. Topology, 10, 55-65 (1971) 